\title{
Sexual dysfunction in old age
}

\author{
André Ludovic Phanjoo
}

The sexual behaviour of older people is more often the target of jocularity or ridicule than the subject of serious scientific research. As a consequence, relatively little is known about the sexual behaviour of the over-65s and such information as is available shows a polarisation according to gender, male sexual behaviour and dysfunction being viewed very much in the light of physical problems, whereas women's sexual behaviour revolves around attitudes towards sexuality and the psychological effects of ageing. This review will address the biological changes associated with ageing, the psychological and social concomitants, the prevalence of sexual dysfunction, its aetiological factors, and the management of common sexual problems including those found in an institutional setting.

\section{Biological changes}

Masters and Johnson described the physiology of coitus as having four components. Excitement, plateau, orgasm and resolution, all of which show age related changes. As ageing increases, desire may not always result in sexual excitement. The triggers for sexual excitement become more specifically sexual and may require intimate body contact and manual stimulation. The intensity of sexual fantasies decreases and it may take a man longer to achieve an erection and following ejaculation more time before an erection is possible. The physiological changes are listed in Box 1. In addition, older men experience anatomical changes, including thinning of the pubic hair, laxity of scrotal tissue, atrophy of the perineal muscles, loss of collagen tissue and occasionally

weight gain. The phase of ejaculation undergoes changes which result in a decline in the intensity of orgasm and in the propulsive force of ejaculation. The volume of the ejaculate may be reduced by $50 \%$. Sex drive and performance vary widely between individuals of the same group and the maintenance of sexual activity depends on factors such as regular sexual activity, the presence of a willing sexual partner, the absence of a major physical illness and the integrity of the relationship.

Age-related changes in women (see Box 2) include thinning of the pubic hair, shrinkage of the labia, thinning of the vaginal mucosa and laxity of the perineal muscle. The thinning of the vaginal mucosa and the reduced lubrication may lead to dyspareunia and bleeding during intercourse. Orgasmic contractions may become painful.

The ageing male experiences a reduction in the activity of the cells of Leydig with an associated drop in testosterone of $0.4-0.8 \%$ per year after the age of 50 . Interested readers are referred to a

Box 1. Physiological changes in ageing
men

Sexual organ atrophy

Diminished testosterone level

Delay in attaining erection

Erection of poor quality

Longer delay in achieving and maintaining a full penile erection

Decline in intensity of orgasm

Decreased hormone levels are associated with reduced desire

André Ludovic Phanjoo is Clinical Director for the Care of the Elderly Unit, Lothian Primary Care Trust (Royal Edinburgh Hospital, Morningside Terrace, Edinburgh EH10 5HF) and Consultant in Charge of the Sexual Problems Clinic. He holds an Honorary Senior Lectureship at the Department of Psychiatry and is Director of the Lothian Memory Treatment Centre. His research interests include depression in the elderly and new therapies for dementia. 
Box 2. Physiological changes in ageing women

Changes are secondary to declining secretion of oestrogen after the menopause

Loss of elasticity in breast tissue and loss of breast dimensions

Cervix and uterus shrink in size

Walls of the vaginal canal atrophy and vaginal length and width decrease

Decrease in vaginal lubrication

Sex steroid starvation may indirectly affect sex drive

comprehensive review of this topic and its relationship to depression by Seidman \& Walsh (1999).

\section{Psychological changes}

Ageism prevails in most Western societies. Social attitudes and beliefs that consider sexual behaviour as inappropriate, repugnant or abnormal in old age will contribute to the curtailment of such behaviour. Older people have a difficult task in coming to terms with the decline in physical attributes, sexual potency and attractiveness. All too commonly they adopt negative attitudes towards their sexuality. A study in Finland (Paunonen \& Hagmann-Laitila, 1990) found that more than half of 50 residents in a nursing home did not consider it proper for older people to have an active sex life; $25 \%$ felt that sexual needs and desires were sinful and shameful. Fewer than a quarter of the respondents were prepared to talk to the staff about sexual matters.

Factors other than social constraints, such as a disturbed relationship, physical fatigue and infirmities, psychological problems and economic worries, will also militate against a regular sex life.

\section{Patterns of sexual behaviour}

A number of factors have been identified as important in the maintenance of sexual activity. Good physical health, the availability of a partner, and a regular and stable pattern of sexual activity earlier in life predict the maintenance of sexual activity in old age. The proportion of sexually active married people declines from $55-60 \%$ in 60 - to $74-$ year-olds to $25 \%$ in the over- $75 \mathrm{~s}$.
Older women were brought up to prize modesty and they are reluctant to complain of sexual problems. A survey relating to urogenital ageing in 2045 people aged between 55 and 85 found that no more than $11 \%$ were affected at any one time (Barlow et al, 1997). Of that group, 73\% were sexually inactive. Of the $11 \%$ affected, only onethird sought professional advice, whereas 36\% resorted to over-the-counter remedies and 33\% did not seek help. Bachmann's (1990) study of 59 healthy post-menopausal women aged 60-70 reported that $66 \%$ were coitally active and $34 \%$ were abstinent. The active group reported more sexual interest, greater sexual satisfaction and on pelvic examination were noted to have less genital atrophy. Little information is available on the sexual behaviour of older women in other cultures. A study on Chilean women reported that $40 \%$ of women over 60 were sexually active (Gramegna et al, 1998). The sexual adaption of women following the loss of their marital partner has been studied (Malatesta et al, 1988). Increasing age of the widow was associated with less unhappiness with loss of marriage-related activities. What the older woman missed most could be expressed as non-sexual heterosexual activity, such as the social company of a man.

Older men report more interest in sex than women do. Most studies find that the most important factors in the maintenance of sexual activity are the availability of the partner and good physical health. A study of 81 healthy married men aged 60-71 with no physical illness, no psychopathology and no marital problems found that $36 \%$ of the sample suffered from erectile failure. Ageing was associated with a decline in the frequency of sexual intercourse and an increase in the frequency of masturbation (Weizman \& Hart, 1987). A sample of 319 men aged 50-80 were surveyed by a postal questionnaire, revealing that physiological potency for men aged 50-59, 60-69 and $70-80$ amounted to $97 \%, 56 \%$ and $51 \%$, respectively (Feldman et al, 1994). Among the oldest men (70-80 years) $46 \%$ reported orgasm at least once a month. Brahler \& Unger (1994), surveying a group of 450 elderly men and women who still had a partner, found that $65 \%$ of people aged $61-70$ and $33 \%$ of those aged over 70 engaged in sexual activity. In a study in Massachusetts, $15 \%$ of $70-$ year-old men were completely impotent and 34\% moderately impotent. The problem was associated with a significant number of physical and medication problems (Feldman et al, 1994).

Few studies have looked at the general population living in the community and relatively little is known about the wider aspects of sexuality, such as intermittent physical contact, masturbation and affection. One recent study reports that $72 \%$ of men 
and $40 \%$ of women over the age of 65 engage in occasional masturbation (Bretschneider \& McCoy, 1988). Other studies have looked at poor self-image and reluctance to talk about sex (White \& Catania, 1982) and sexual difficulties associated with dementia (Haddad \& Benbow, 1993).

\section{Types of sexual dysfunction}

A recently published sexual dysfunction survey in the USA involved 1410 men and 1749 women aged 18-59, of whom 79\% took part. In women, 32\% complained of a lack of interest in sex, $26 \%$ of an inability of achieve orgasm and $16 \%$ of pain during intercourse. Of the men, 31\% complained of premature ejaculation, $18 \%$ of performance anxiety and, in the 50- to 59-year-old age group, erectile failure was present in $18 \%$. Low sexual desire was associated with general unhappiness, and sexual dysfunction was strongly associated with unsatisfactory personal relationships (Laumann et al, 1999).

The DSM-IV (American Psychiatric Association, 1994) proposes seven categories of sexual dysfunctions. The first three are related to disorders of desire, arousal and orgasm. Categories four to six define sexual problems associated with pain, physical illness, and drugs and alcohol, and category seven defines disorders that are not otherwise specified. In older men, erectile failure is by far the most frequently encountered problem, whereas in older women loss of sexual interest and motivation is the main problem.

Older people do not seek help for their sexual problems for a variety of reasons. In my Sexual Problems Clinic in Edinburgh, out of a total of 3340 people seen over 25 years, only 54 patients were over the age of 65 and all of them were male (see Table 1).

\section{Aetiology}

Numerous factors contribute to a varied and complex aetiology. They include the biological changes of ageing, negative cultural expectations, medical or surgical problems, the effects of drugs, and mental illnesses such as depression, psychosis and dementia.

\section{Physical disorders}

Ageing is associated with an increase in the prevalence of chronic illnesses, in the number of
Table 1. Patients aged over 65 years seen at the Sexual Problems Clinic in Edinburgh (1974-1999) (total $n=54$, all male)

Dysfunction

Number presenting

Erectile failure 36

Loss of sexual interest

Paedophilia

Gender dysphoria

2

prescribed drugs and also in the likelihood of surgical procedures which have distressing physical and psychological sequelae. In addition, there may be damage to the vascular integrity of the genitalia and functional limitation of the pulmonary and cardiovascular systems. Illnesses that contribute to sexual problems in old age include cardiovascular problems, particularly hypertension, peripheral vascular disease, diabetes mellitus, renal failure, cancer, arthroses and neurological problems (Feldman et al, 1994) and the sequelae of various operations such as prostatectomy, hysterectomy, mastectomy and various ostomies. The effects of prostatectomy on sex function have been well studied. According to one study (Thorpe et al, 1994), prostatectomy is associated with major erectile failures in $12 \%$ and absent ejaculation in $24 \%$ of cases. The sexual dysfunction found in prostate cancer patients is largely due to the effects of treatment, with radical prostatectomy carrying the highest risk (Helgason et al, 1996). Finasteride, which is used in prostatic enlargement, is associated with loss of libido, erectile failure and reduced volume of ejaculate in 5-6\% of men (Gormley et al, 1992).

Illnesses that result in disability, such as stroke and dementia, invariably lead to important changes in sexual behaviour (Boldrini et al, 1991). There is little evidence that post-stroke patients are routinely given an opportunity to discuss their sexuality openly with their physician. Changes in sexuality after a stroke are often due to a change in role function because of the increased dependency of the afflicted partner (Burgener \& Logan, 1989).

\section{Mental disorders}

The most common mental disorders affecting older people are depression, dementia, delusional disorders and delirium. Sexual behaviour may change significantly in depression (Feldman et al, 1994) and dementia (Haddad \& Benbow, 1993). Delusional disorder may be associated with pathological jealousy, which may cause severe distress to the other partner as well as endangering 
his or her well-being. Sexual problems may be further compounded in this group of patients by the use of psychoactive agents.

\section{Substance-induced sexual dysfunction}

The effects of chronic alcohol misuse on sexual function are well recognised and will not be discussed in detail here (Wagner \& Jensen, 1981). In small doses, alcohol acts as an anxiolytic and may improve performance, but larger doses will result in retarded orgasm and erectile failure. Drugs like cocaine and amphetamines increase sexual interest and activity initially because of their enhancing effects on mood and energy, but the long-term effects are usually detrimental.

\section{Pharmacological agents and sexual dysfunction}

There is a significant literature on the sexual sideeffects of antidepressant drugs - one review (Ellison, 1998) cites 118 references. All types of antidepressants, including monoamine oxidase inhibitors, tricyclics and selective serotonin reuptake inhibitors (SSRIs) are implicated in a variety of sexual problems affecting libido, arousal and ejaculation. Depression per se is also associated with sexual problems and it is, therefore, important for clinicians to enquire carefully as to what specific phase of the sexual response cycle is affected. There are now specific diagnostic criteria for antidepressant-induced sexual dysfunction in DSM-IV, subsumed under category 292.89. It is likely that as up to $50 \%$ of patients treated with SSRIs may develop sexual dysfunction, mainly related to delayed or absent ejaculation or orgasm. These drugs may also be involved in erectile failure. Once an antidepressant-induced sexual dysfunction has been established, appropriate treatment may involve a reduction in the offending medication, a 'drug holiday' or a change to a different antidepressant. The antidepressants that are least likely to affect sexuality are nefazodone, mirtazapine and reboxetine. Occasionally, patients treated with SSRIs may report increases in sexual desire. The drugs implicated here are trazodone, bupropion and, much less frequently, venlafaxine, paroxetine and fluoxetine. Trazodone is the drug most frequently associated with priapism (Warner et al, 1987). This problem has also been reported with phenothiazines, butyrophenones and benzisoxazoles (Patel et al, 1996).

Antipsychotic drugs, however, have a predominantly depressant effect on sexual function through their effects on the dopaminergic system.
Non-psychotropic medication may also have a negative influence on sexuality. Anticonvulsants, antihypertensives, $\mathrm{H}_{2}$ blockers and thiazide diuretics (O'Keefe \& Hunt, 1995) as well as cimetidine, digoxin and metoclopramide (Guay, 1995) are known to cause erectile failure. A full discussion of this topic can be found in Ellison (1998).

\section{Management of sexual dysfunction}

Assessment of a sexual dysfunction requires a careful history from the client with a corroborative history from the spouse/partner. Hawton (1985) has summarised the important features of a sexual history as:

(a) establishing a language that is mutually comfortable;

(b) using a mixture of closed and open questions;

(c) getting an accurate description of what is actually wrong;

(d) taking a longitudinal perspective;

(e) interviewing both partners jointly and separately; and

(f) taking a medical history including a complete list of drugs that the client is taking.

Many older couples need accurate information about the physiological changes associated with ageing. Patient education is therefore an important part of the management of sexual dysfunctions (Bachmann, 1990). The range of treatments available comprises psychosexual counselling, hormonal therapy, drug treatment, mechanical devices and vascular surgery (Box 3$)$.

\section{Psychosexual counselling}

This is an important aspect of any management plan since sexual dysfunctions, whatever the underlying cause, usually have a psychological overlay. It is,

Box 3. Treatment options

Basic education and advice

Psychosexual counselling

Hormonal therapy

Drug treatment

Mechanical devices

Vascular surgery 
therefore, recommended that pharmacological treatment be combined with counselling.

\section{Hormonal treatment}

The use of testosterone supplements to treat the viropause (male menopause) is controversial. The proportion of older men with a significantly reduced testosterone level is low. Large epidemiological studies have shown that total testosterone remains steady until the age of 50 and then declines by approximately $0.4-0.8 \%$ per year (Gray et al, 1991). Administration of testosterone to eugonadal men leads to a small increase in sexual interest but it is no more effective than placebo in improving sexual dysfunction (Schiavi et al, 1997). On the other hand, there are numerous reports in the literature attesting to the benefits of androgen supplements in hypogonadal men. Libido, arousal and ejaculatory capacity all show improvements (Morley et al, 1993). The empirical use of exogenous androgens in eugonadal elderly men should not be encouraged as such treatments potentially could induce polycythaemia, worsen coronary artery disease and exacerbate benign prostatic hypertrophy (Seidman \& Walsh, 1999).

In women the use of hormone replacement therapy (HRT) is widespread although still controversial. It reverses the physiological changes associated with sex steroid starvation. Post-menopausal women who suffer from loss of libido in addition to the physiological changes associated with oestrogen deficiency may benefit from the gonadomimetic tibolone.

\section{Drug treatment (Box 4)}

Oral agents

A variety of drugs have been used in the treatment of male sexual dysfunctions, including yohimbine, phentolamine, apomorphine, bromocriptine and trazodone. The availability of sildenafil has had a tremendous impact on the management of erectile failure. Sildenafil is a phosphodiesterase inhibitor and its success has tended to eclipse other forms of drug treatment. Sildenafil has been shown to be an effective treatment both in non-organic erectile failure $(89 \%$ response at $50 \mathrm{mg})$ and also in the organic impotence secondary to diabetes mellitus, hypertension and neurological disorders.

Clinical experience suggests that men with psychogenic impotence respond well to $50 \mathrm{mg}$ sildenafil, whereas $100 \mathrm{mg}$ is more effective in organic impotence. The drug is rapidly absorbed, although the ingestion of food will delay absorption. It is safe and has relatively minor side-effects, the
Box 4. Drug treatment

Yohimbine

Oral phentolamine

Apomorphine sublingual tablets

Sildenafil

Intracaversonal injection of vasoactive drugs

Intraurethral insertion of prostaglandin (MUSE)

Use of vasoactive cream

main ones being headaches, flushing, dyspepsia, nasal congestion and abnormal vision. It is contraindicated in patients taking nitrates because of the danger of provoking severe hypotension. The possibility of a synergistic action between sildenafil and non-nitrate antihypertensive medication has recently been raised (Cohen, 2000), although an editorial reply (Kloner \& Siegel, 2000) refuted this suggestion. Not every patient will respond to sildenafil and it is very unlikely that patients with severe vasculogenic impotence or cavernosal fibrosis will show a response.

Oral phentolamine has been tried in mild erectile failure and there is some evidence that the drug is beneficial in about $50 \%$ of cases (Goldstein et al, 1998). More recently, it has been used in female sexual arousal disorder in menopausal women. The results indicate a mild positive effect of phentolamine across all measures of arousal (Rosen $e t$ al, 1999). There are also anecdotal reports of the value of sildenafil in arousal disorders in women.

Premature ejaculation has been mostly treated by psychological methods such as the squeeze technique or the stop-start method. Over the years, there have been sporadic reports on the effectiveness of certain drugs such as thioridazine, clomipramine and the SSRIs. A recent study (Strassberg et al, 1999) suggests that clomipramine $25 \mathrm{mg}$ taken as needed as little as four hours prior to sexual activity is of value in treating premature ejaculation.

\section{Intraurethral drugs}

Prostaglandin $\mathrm{E}_{1}$ is used as an intraurethral pellet in a preparation known as MUSE (medicated urethral system for erection). In a large study involving 1511 men suffering from impotence due to a variety of causes, the improvement rate was $66 \%$. Over an extended period, however, the results were less impressive (Padma-Nathan et al, 1997). 


\section{Topical creams}

Most topical creams that have been used in erectile failure are smooth muscle relaxants and vasodilators. These include glyceryl trinitrate, minoxidil, prostaglandin $\mathrm{E}_{1}$, as well as a triple cream containing aminophylline, isosorbide dinitrate and codergocrine mesylate. Whereas the results with most of these have been disappointing, one study using the triple cream reported a $60 \%$ improvement rate (Gomaa et al, 1996). A recent study (Le Roux \& Naude, 1999) was unable to reproduce the successful results reported earlier and they found no difference between the active cream and the placebo cream.

\section{Intracorporal injection therapy}

Since the early 1980s, a variety of drugs have been used effectively by intracorporal injection in the treatment of erectile failure. Papaverine, phentolamine, prostaglandin $\mathrm{E}_{1}$ and moxisylyte, among others, have been tried. The most frequently used drug is now prostaglandin $E_{1}$ because of its favourable side-effect profile relative to the earlier preparations, which occasionally cause penile pain, bruising, hypotension, flushing, fibrosis and priapism. Although the response rate is about $65 \%$, some studies have shown that there is a drop-out rate of about $25-40 \%$ over a two-year period. For a fuller discussion of this topic, see Eardley \& Sethia (1998).

\section{Mechanical devices}

There are a number of these, the most popular being the vacuum constriction devices. Their use increased during the 1980s and for some time they were the most popular form of treatment for erectile failure, particularly in older men. In a large series of 1517 patients treated by this method, $92 \%$ were able to have an erection and $77 \%$ were able to have sexual intercourse (Witherington, 1989). There are conflicting data about the effectiveness of these devices when used over an extended period of time. The pros and cons of this type of treatment are fully discussed by Eardley \& Sethia (1998).

Penile implants have been in use for over 40 years and different types of prostheses are available. Vascular surgery is occasionally used when arterial or venous abnormalities have been clearly demonstrated.

\section{Sexual behaviour in institutional settings}

A significant number of older people who require institutional care suffer from either physical or mental impairment of such a degree that it is no longer safe for them to live independently. In an institutional setting, sexual behaviour becomes linked to questions relating to privacy, attitudes of the nursing staff, and cultural expectations of other residents. Sexual disinhibition may be present in about $7 \%$ of cognitively impaired older people. There is an ongoing conflict between protecting patients' dignity and the patients' desire to fulfil their sexual needs. Nursing home staff as well as visitors or family may feel embarrassment when confronted with the sexuality of older people. Sometimes this may lead to older people being treated like children and being forbidden to have any sexual outlet. This, in turn, may provoke anger and frustration in the patients.

People with dementia may become less interested or more interested in sex. They may become more childlike and less subtle in their sexual demands. They may undress and/or masturbate in front of other residents or visitors. They may seek to engage other residents sexually. There is a conflict between supporting autonomous decision-making and protecting residents from abuse and exploitation. The resolution to the conflict is obviously easier if one or both partners, despite cognitive impairment, is aware of the implications of the relationship and able to decide whether they want to continue with the relationship or not. It is much more difficult if one or both partners is so cognitively impaired that they have no awareness of what they are doing. In such situations, it is important that the staff have the opportunity of discussing each client individually and involving their relatives/carers instead of adopting a blanket approach using pharmacological agents to contain such behaviour. There may be situations where, other methods having failed, it may be necessary to use drugs in order to inhibit undesirable or socially unacceptable sexual behaviour. Various agents have been tried and good results have been reported with SSRIs, anti-androgen therapy and oestrogen. The use of the oestrogen patch is an excellent treatment in the management of elderly men with dementia who suffer from sexual disinhibition. There are also isolated reports on the successful use of antiandrogens in elderly women with dementia who exhibit compulsive and antisocial sexual behaviour.

\section{Conclusion}

Very few older people consult professionals because of sexual dysfunction. Epidemiological data are sparse and studies are usually not comparable 


\section{Box 5. Key points}

Sexual problems are common in old age, but older people are reluctant to seek help

Sexual problems are associated with the biological changes of ageing, physical illness, the effect of various drugs and the negative sociocultural ethos

Older people need basic education and advice concerning their adjustment to age-related physiological changes in their sexuality

Pharmacological treatment is highly effective in erectile failure regardless of aetiology

Problems of sexual attitude and behaviour in institutions should be discussed openly

because of the differences in the sample groups. As life span increases and older people become healthier, they will have increasing expectations regarding their sexuality. Clinicians and professional helpers need to be aware of such problems, be ready to educate and advise older people on how best to adapt to physiologically altered responses and, in some cases, treat them by psychosexual counselling or chemotherapy as well as alleviate any physical problems.

\section{References}

American Psychiatric Association (1994) Diagnostic and Statistical Manual of Mental Disorders (4th edn) (DSM-IV). Washington, DC: APA

Bachmann, G. A. (1990) Sexual issues at menopause. Annals of the New York Academy of Sciences, 592, 87-94.

— \& Leiblum, S. R. (1991) Sexuality in sexagenarian women. Maturitas, 13, 43-50

Barlow, D. H., Cardozo, L. D., Francis, R. M., et al (1997) Urogenital ageing and its effect on sexual health in older British women. British Journal of Obstetrics and Gynaecology, 104, 87-91.

Boldrini, P., Basaglia, N. \& Calanca, M. C. (1991) Sexual changes in hemiparetic patients. Archives of Physiological and Medical Rehabilitation, 72, 202-207.

Brahler, E. \& Unger, U. (1994) Sexual activity in advanced age in the context of gender, family status and personality aspect. Zeitschrift für Gerontologie, 27, 110-115.

Bretschneider, J. D. \& McCoy, N. L. (1988) Sexual interest and behaviour in healthy 80-102 year olds. Archives of Sexual Behaviour, 17, 109-129.

Burgener, S. \& Logan, G. (1989) Sexuality concerns of the post stroke patient. Rehabilitation Nursing, 14, 178-181.

Cohen, J. S. (2000) Sildenafil and nonnitrate antihypertensive medications (letter). Journal of the American Medical Association, 283, 201-202.

Eardley, I. \& Sethia, K. (1998) Erectile Dysfunction. London: MosbyWolfe.
Ellison, J. M. (1998) Antidepressant induced sexual dysfunction. Review, classification and suggestions for treatment. Harvard Review of Psychiatry, 6, 177-189.

Feldman, H. A., Goldstein, I., Hatzichristou, D. G., et al (1994) Impotence and its medical and psychological correlates: results of the Massachusetts male ageing study. Journal of Urology, 151, 54-61.

Goldstein, I. \& the Vasomax Study Group (1998) Efficacy and safety of oral phentolamine for the treatment of minimal erectile dysfunction. Journal of Urology, 159, abstract 919.

Gomaa, A., Shalaby, M. \& Osman, M. (1996) Topical treatment of erectile dysfunction. British Medical Journal, 312, 1512-1515.

Gormley, G. J., Stoner, E., Bruskewitz, R. C., et al (1992) The effects of finasteride in men with benign prostatic hyperplasia. New England Journal of Medicine, 327, 1185-1191.

Gramegna, G., Blumel, J. E., Roncagliolo, M. E., et al (1998) Patterns of sexual behaviour in Chilean women. Revista Medica de Chile, 126, 162-168.

Gray, A., Berlin, J. A., McKinlay, J. B., et al (1991) An examination of research design effects on the association of testosterone and male ageing: results of a metaanalysis. Journal of Clinical Epidemiology, 7, 671-684.

Gregoire, A. \& Pryor, J. (eds) (1993) Impotence: An Integrated Approach to Clinical Practice. Edinburgh: Churchill Livingstone.

Guay, A. T. (1995) Erectile dysfunction - are you prepared to discuss it? Postgraduate Medicine, 97,127-143.

Haddad, P. M. \& Benbow, S. M. (1993) Sexual problems associated with dementia. International Journal of Geriatric Psychiatry, 8, 547-551; 631-637.

Hawton, K. (1985) Sex Therapy: A Practical Guide. Oxford: Oxford University Press

Helgason, H. R., Adolfsson, J., Dickman, P., et al (1996) Sexual desire, erection, orgasm and ejaculatory functions and their importance to elderly Swedish men: a population-based study. Age and Ageing, 25, 285-291.

Kloner, R. A. \& Siegel, R. (2000) Sildenafil and nonnitrate antihypertensive medications: Editorial reply. Journal of the American Medical Association, 283, 202.

Laumann, E. O., Paik, A. \& Rosen, R. C. (1999) Sexual dysfunction in the USA: prevalence and predictors. Journal of the American Medical Association, 281, 537-544.

Le Roux, P. J. \& Naude, J. H. (1999) Topical vasoactive cream in the treatment of erectile failure: a prospective, randomised, placebo-controlled trial. British Journal of Urology, 83, 810-811.

Malatesta, V. J., Chambless, D. L., Pollack, M., et al (1988) Widowhood, sexuality and ageing: a life span analysis. Journal of Sex and Marital Therapy, 14, 49-62.

Morley, J. E., Perry, H. M. \& Kaiser, F. E. (1993) Effects of testosterone replacement in old hypogonadal males: a preliminary study. Journal of the American Geriatrics Society, 41, 149-152.

O'Keefe, M. \& Hunt, D. K. (1995) Assessment and treatment of impotence. Medical Clinics of North America, 79 414-435.

Padma-Nathan, H., Hellstrom, W. \& Kaiser, F. E. (1997) Treatment of men with erectile dysfunction with transurethral Alprostadil. New England Journal of Medicine, $336,1-7$.

Patel, A. G., Mukherji, K. \& Lee, A. (1996) Priapism associated with psychotropic drugs. British Journal of Hospital Medicine, 55, 315-319.

Paunonen, M. \& Hagmann-Laitila, A. (1990) Sexuality and the satisfaction of sexual needs: a study on the attitudes of aged nursing home clients. Scandinavian Journal of Caring Sciences, 4, 163-168.

Rosen, R. C., Philips, N. A., Gendrano, N. C., et al (1999) Oral phentolamine and female sexual arousal disorder: a pilot study. Journal of Sex and Marital Therapy, 25, 137-144.

Schiavi, R. C., White, D. \& Mandeli, J. (1997) Effects of testosterone adminstration on sexual behaviour and mood in men with erectile dysfunction. Archives of Sexual Behavior, 26, 231-241.

Seidman, N. J. \& Walsh, B. T. (1999) Testosterone and depression in ageing men. American Journal of Geriatric Psychiatry, 7, 18-33. 
Strassberg, D. S., De Gouveia Brazao, C.A., Rowland, D. L., et al (1999) Clomipramine in the treatment of rapid ejaculation. Journal of Sex and Marital Therapy, 25, 89-101.

Thorpe, A. C., Cleary, R., Coles, J., et al (1994) Written consent about sexual function in men undergoing transurethral prostatectomy. British Journal of Urology, 74, 479-484.

Wagner, G. \& Jensen, S. B. (1981) Alcohol and erectile failure. In Impotence. Physiological, Psychological, Surgical Diagnosis and Treatment (eds G. Wagner \& R. Green). New York: Plenum.

Warner, M. D., Peabody, C. A., Whiteford, H. A., et al (1987) Trazodone and priapism. Journal of Clinical Psychiatry, 48, 244-245.

Weizman, R. \& Hart, J. (1987) Sexual behaviour in healthy married elderly men. Archives of Sexual Behavior, 16, 3944.

White, C. B. \& Catania, J. A. (1982) Psychoeducational intervention for sexuality with the aged, family of the aged, and people who are working with the aged. International Journal of Aging and Human Development, 15, $121-138$.

Witherington, R. (1989) Vacuum constriction device for management of erectile impotence. Journal of Urology, 141 $320-322$.

\section{Multiple choice questions}

1. The following treatments have been shown to be effective in eugonadal men with erectile dysfunction:
a vacuum constriction devices
b testosterone
c benzodiazpines
d MUSE (medicated urethral system for erection)
e sildenafil.

2. Physical changes in ageing women include:
a thinning of the vaginal walls
$\mathrm{b}$ increased vaginal lubrication
c shrinkage of the labia
d a shorter period to become sexually aroused
e painful contractions during orgasm.

3. The preservation of an active sex life in old age is related to:
a good physical health
b readiness to seek medical advice
c the availability of a partner
d a regular and stable pattern of sexual activity earlier in life
e economic affluence.

4. Reduced sexual activity in older women is associated with:
a inability to achieve orgasm
b pain during intercourse
c lack of interest in sex
d performance anxiety
e the use of hormone replacement therapy.

5. Sildenafil is contraindicated in the following patients:

a those on regular nitrates

$b$ those on insulin

c those taking antidepressants of the SSRI type

d those with sickle cell anaemia

e those on benzodiazepines.

\section{MCQ answers}

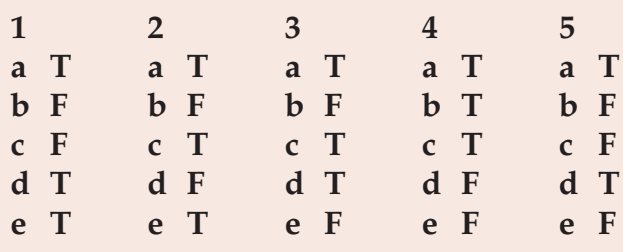

